Small angle detectors for study diffractive processes with CMS

This content has been downloaded from IOPscience. Please scroll down to see the full text. 2014 JINST 9 C10032

(http://iopscience.iop.org/1748-0221/9/10/C10032)

View the table of contents for this issue, or go to the journal homepage for more

Download details:

IP Address: 131.225.23.169

This content was downloaded on 08/12/2014 at 17:12

Please note that terms and conditions apply. 


\section{Small angle detectors for study diffractive processes with CMS}

M. Albrow, ${ }^{a}$ A.J. Bell, ${ }^{b}$ D. d Enterria, ${ }^{b}$ R. Hall-Wilton, ${ }^{b}$ S. Los, ${ }^{a}$ N. Mokhov, ${ }^{a}$

M. Murray, ${ }^{c}$ A. Penzo, ${ }^{d}$ S. Popescu, ${ }^{e}$ A. Ronzhin, ${ }^{a}$ V.D. Samoylenko, ${ }^{f, 1}$ A. Sobol ${ }^{f}$ and $\mathbf{G}$. Veres ${ }^{b}$

${ }^{a}$ FNAL, Batavia, IL 60510, U.S.A.

${ }^{b}$ CERN, CH-1211 Geneva 23, Switzerland

${ }^{c}$ Kansas State University, Manhattan, KS 66506, U.S.A.

${ }^{d}$ INFN, Sezione di Trieste, I-34127 Trieste, Italy

eIFIN-HH, Atomistilor st., Bucharest, 407, Romania

${ }^{f}$ IHEP, 1 Nauki Sq., Protvino, 142281, Russia

E-mail: Vladimir.Samoylenko@ihep.ru

ABSTRACT: The approach and detectors for diffractive physics based on two current projects Forward Shower Counter (FSC) and Proton Precision Spectrometer (PPS) are presented.

FSC system consists of six $(3+3)$ Stations of scintillator counters, which surround closely the beam pipes along $59 \mathrm{~m}<|z|<140 \mathrm{~m}$ from IP5 on both plus $(+)$ and minus $(-)$ sides. These will detect showers from very forward particles with rapidity $7.5<|\eta|<10$ interacting in the beam pipe and surrounding material. FSC allow measurements of single diffraction: $p+p \rightarrow p+G+X$ (where $G$ is rapidity gap) for lower masses and double diffraction $p+p \rightarrow X+G+X$ with a large central rapidity gap. The counters can also be used for beam real-time monitoring and will make an invaluable contribution to the understanding of the background environment and its topology.

PPS is designed for study the central exclusive production $p p \rightarrow p+X+p$, where the + signs denote the absence of hadronic activity (that is, the presence of a rapidity gap) between the outgoing protons and the decay products of the central system $X$. The precise measurement of the kinematical parameters of the outgoing protons enables to study the properties of the central state $X$. In PPS part we consider the detector for high precision timing of these protons - QUARTIC. It consists of L-shape bars with quartz or sapphire radiator. The time resolution of the QUARTIC prototypes achieved $\approx 10 \mathrm{ps}$.

KEYWORDS: Timing detectors; Instrumentation and methods for time-of-flight (TOF) spectroscopy; Cherenkov and transition radiation

\footnotetext{
${ }^{1}$ Corresponding author.
} 


\section{Contents}

1 Introduction $\quad 1$

2 Forward Shower Counter $\quad 3$

3 Proton Precision Spectrometer $\quad 4$

4 Conclusion $\quad 6$

\section{Introduction}

We consider the approach and detector for diffractive physics based on two current projects Forward Shower Counter (FSC) and Proton Precision Spectrometer (PPS).

A major goal of the early programme of Forward Physics is the measurement of the main characteristics of diffractive interactions [1]. Different behaviours of the diffractive cross sections are predicted for asymptotic behaviours of the total cross section $\sigma_{\text {tot }}[2,3]$. It is therefore important to study diffractive dissociation processes at the LHC. To constrain the parameters of the models of soft diffraction one needs to make measurements at LHC energies of the single diffractive dissociation cross section for low masses, $\sigma_{\mathrm{SD}}$ (low masses), and of central diffractive production $\frac{d \sigma}{d \eta_{1} d \eta_{2}}$, where $d \eta_{1}$ and $d \eta_{1}$ define the pseudorapidity $(\eta=-\ln \tan \theta / 2)$ range of the central system. Single diffractive dissociation is the process $p+p \rightarrow p+p^{*} \rightarrow p+X$, where $X$ is a system of particles with typically $M(X) \sim$ few $\mathrm{GeV} / \mathrm{c}^{2}$ and the + sign represents a large ( $>3$ units) rapidity gap, meaning no hadrons in pseudorapidity between the outgoing proton and the diffractive system $X$. This physics is not possible with the central detectors, as the hadrons coming from the fragmentation of $X$ have forward (longitudinal) momenta $\sim \mathrm{TeV} / \mathrm{c}$ and transverse momenta $p_{T}<1 \mathrm{GeV} / \mathrm{c}$.

None of the major LHC detectors (ALICE, ATLAS, CMS, and LHCb) have the coverage necessary to measure forward rapidity gaps. CMS has exellent hermeticity at low rapidity region $\eta$, in forward direction CMS coverage is extended with different additional detectors - HF + CASTOR + ZDC (and TOTEM [4]). But there is a gap in high $\eta$ region (figure 1). The FSC System is designed to select events with this key rapidity. At present, without detecting diffractively scattered protons and without the possible of detecting very forward rapidity gaps we are unable to distinguish events dominated by diffraction from non-diffractive events. As most of the pile-up events will have forward particles giving showers in the FSC, they can be effectively vetoed at the Level 1 trigger. Showers will usually give a large pulse height, many times that of a minimum ionizing particle (MIP), and are easily discriminated from noise. For instance, diffractive excitation one would require all the counters on one side (in logical OR) to be consistent with noise. The FSC could also serve as a luminosity monitor by measuring the fraction of bunch crossings with no inelastic collisions, as well as monitoring beam conditions [5]. 


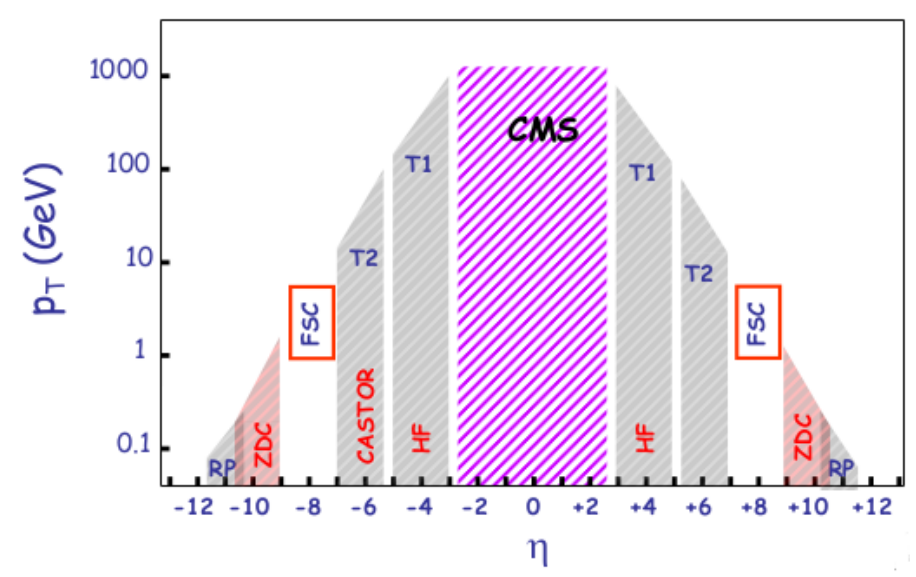

Figure 1. FSC covers a gap in rapidity $\eta$ in the forward calorimeters (HF, CASTOR) and the very forward (ZDC, TOTEM).

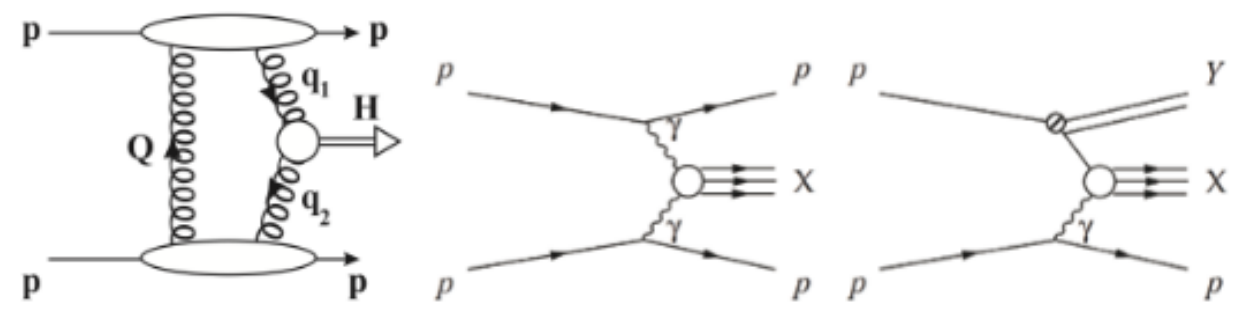

Figure 2. The relevant diagrams accessible to study with PPS. Left: Central Exclusive Production (CEP), $p p \rightarrow p+H+p$; middle: exclusive photon-photon production, $p p \rightarrow \gamma \gamma \rightarrow p+X+p$; right: photon-proton production, $p p \rightarrow p+X+Y$.

The Proton Precision Spectrometer (PPS) forward proton tagging capability can enhance the ability of the CMS detector to carry out the primary physics program of the LHC in various sectors and extensions of the Standard Model. By central exclusive production we refer to the process $p p \rightarrow p+\phi+p$, where the + signs denote the absence of hadronic activity (that is, the presence of a rapidity gap) between the outgoing protons and the decay products of the central system $\phi$. The final state therefore consists solely of the two outgoing protons, which we intend to detect in the spectrometer, and the decay products of the central system, which will be detected in the central CMS detectors. The main physics motivation is the production and study of Higgs bosons, but there is also a rich and more exotic physics menu that includes the production of many kinds of supersymmetric particles, other exotica, and indeed any new object which has $0^{++}$(or $2^{++}$) quantum numbers and couples strongly to gluons or to photons. For instance the CEP process is illustrated for Higgs boson production in figure 2 (left). Precision proton tracking and timing detectors in the very forward region on both sides of CMS enables a fundamentally different means for analyzing high-luminosity $p p$ collisions. In order to detect the outgoing intact, but off-momentum protons from the CEP process, small tracking detectors must be placed close to the LHC beam at a few hundred meters away from the interaction point. By exploiting the dipoles used to separate the proton beams, this creates a high precision spectrometer that can accurately measure the momentum of the protons. The tracking and timing detectors are housed inside moving beam pipe sections, so 

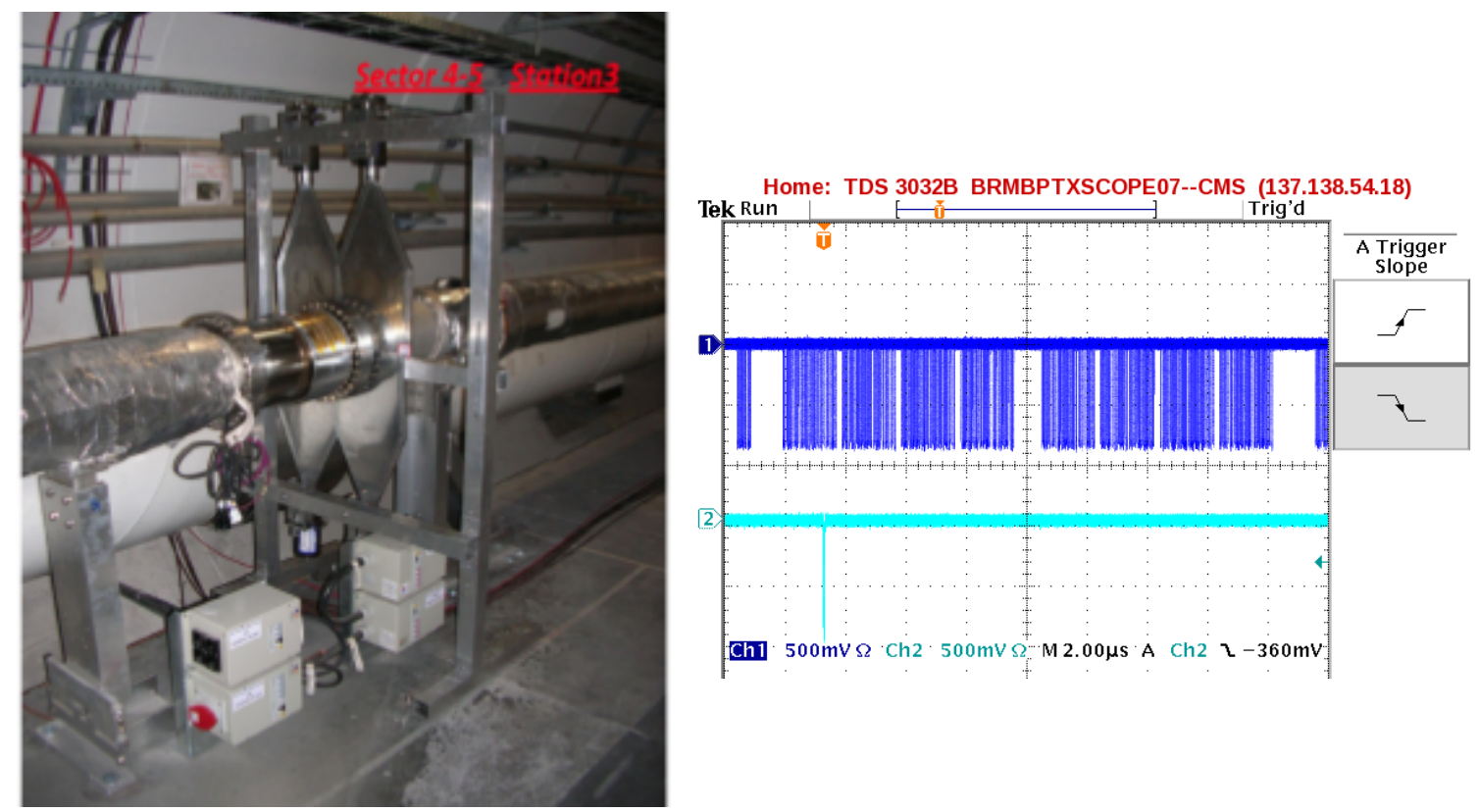

Figure 3. The FSC Station 3 in LHC tonnel (left); LHC bunch train detected by FSC Station, triggering from LHC synchropulse (right).

that the detectors can be parked away from the beam during injection, and then brought close to the beam during collisions. Silicon 3D detectors consist of an array of columnar electrodes (radius $\approx 5 \mu \mathrm{m}$ ) of both doping types which penetrate entirely through the detector bulk, perpendicularly to the surface [6]. 3D detectors are emerging as one of the most promising technologies for the innermost layers of tracking devices for the foreseen upgrades of the LHC [7]. As an additional feature, 3D technology is suitable for manufacturing detectors with active edges, where the insensitive edge region can be reduced compared to hundreds of $\mu \mathrm{m}$ for standard planar detectors. So we can see that these two projects are supplement each other for diffractive processes.

\section{Forward Shower Counter}

FSC is the set of the scintillation counters, closely surrounding the beam pipes with $59<|z|<$ $140 \mathrm{~m}$ from LHC IP5 on both plus (+) and minus ( - ) sides. They do not detect primary particles directly from the $p p$ collisions, but showers produced by small angle and high energy $(\sim \mathrm{TeV})$ particles that hit the beam pipes and surrounding material. The counters will cover $7<|\eta|<11$, where $\eta$ is the pseudorapidity, depending on the particle type and $p_{T}$. Also they can be used effectively as a pile-up veto in the level 1 trigger for single diffraction, especially for hard diffraction (W, Z, dijets) and central exclusive production.

The FSC system consists of scintillation counters with size $20 \times 25 \times 2 \mathrm{~cm}^{3}$. The first two stations involves two (top and bottom) counters, and the last ones - from four counters, figure 3 left. The scintillator was produced by Eljen Company [8] and the PM used are XP2020. The MIP signal is about 30 photoelectrons. This ensures the high effeciency performance for physical purposes. The FSC will have several other uses, including real-time beam halo monitoring of 
both incoming and outgoing beams, which are both in the same pipe at these locations, like it was performed in LHCb [9]. Measurements can be done of the rates with one beam in the machine, and of correlations rates with other monitors. The LHC bunch train detected by FSC is shown in figure 3 right.

\section{Proton Precision Spectrometer}

The PPS physics program requires standard high luminosity running with mean pile-up $\mu=30-40$ inelastic collisions per bunch crossing. Most bunch crossings with two forward protons and a high mass central state $X$ are not the desired $p X p$ collision events, but are pile-up background. As the signal events are exclusive, and if $X$ is fully measured, these pile-up backgrounds are reduced by four-momentum conservation. Fortunately pile-up background can be further reduced by a factor $\approx 20$ by measuring the time difference between the two protons, as proposed in [10]. The time difference $\delta_{t}$ of the two oppositely-directed protons arrival at far detectors, hundreds of meters along the beam pipes, gives a measure of the collision point $z_{p p}$ if they came from the same collision. If the intrinsic resolution of the timing detector is $\sigma_{t}$, the resolution on the time difference is $\sigma(\delta t)=\sqrt{2} \sigma_{t}$, and with $\sigma_{t}=10(15) \mathrm{ps}$ we have $\sigma\left(z_{p p}\right)=2.12 \mathrm{~mm}(3.18 \mathrm{~mm})$.

Requirements on the timing detectors (including their readout electronics), with area only $\approx 2 \mathrm{~cm}^{2}$, are

- a resolution $\sigma(t) \approx 10 \mathrm{ps}$

- active within about $200 \mu \mathrm{m}$ of the beam pipe, to minimize loss of acceptance;

- sufficiently radiation hard;

- capability of being read out every 25 ns without afterpulses;

- with a lifetime requiring replacement not more often than once per year.

We plan to use multi-element Cherenkov counters based on UVT quartz (fused silica) bars [11]. This detector is called QUARTIC for QUARtz TIming Cherenkov.

A first QUARTIC design has long bars inclined at the Cherenkov angle, $\theta_{\mathrm{Ch}} \approx 48^{\circ}$, read out by large area single-anode or multi-anode MicroChannel Plate-PhotoMultipliers, figure 4. A potential weakness of MCP-PMTs is that the photocathode gets damaged by positive ion feedback, which limits their life to typically $10^{14}$ photoelectrons, which would be only weeks in the PPS environment. To solve this problem we proposed novel geometry - L shape bar shown in figure 5. It combines the virtues of having the Cherenkov radiator bar parallel to the beam (with $100 \%$ of the radiated light from protons moving parallel to the bar axis being trapped along the bar) and having the individual photodetector far from the beam. The bar is L-shaped with a $90^{\circ}$ corner. If the surfaces are perfect, no light is refracted out and it all reaches the end of the light guide (no mirrors!), except the light emitted in the plane perpendicular to the light guide (LG) bar. Since $n(\lambda)>\sqrt{2}$ so that $\theta_{\mathrm{Ch}}>45^{\circ}$ as it is for quartz, the light that passes up the LG bar has an angle with respect to the surface that is $<45^{\circ}$, less than the critical angle, and total reflection is maintained. This means that the path length of the light and number of reflections per unit length are all less than in the radiator 

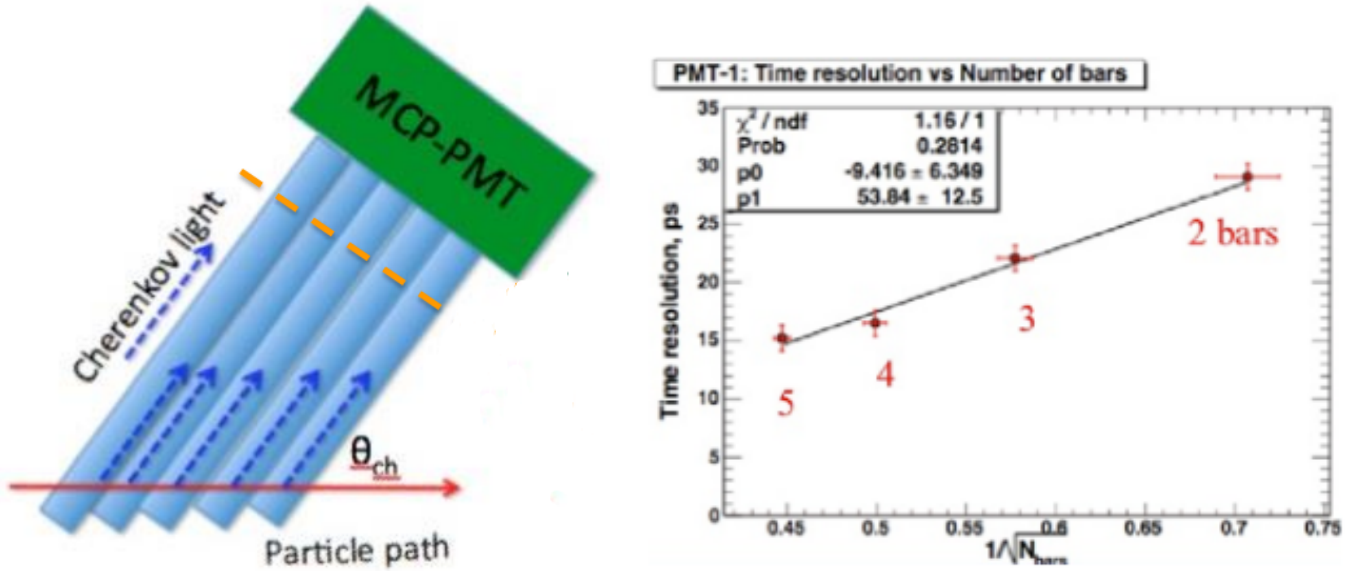

Figure 4. Arrangement of an angled-bar QUARTIC on a PHOTONIS MCP-PMT (figure courtesy of A. Brandt) (left); the time resolution of angled bars on the PMT240, for different numbers of installed bars, plotted versus $1 / \sqrt{N}$, showing the expected $\sqrt{N}$ improvement (right).

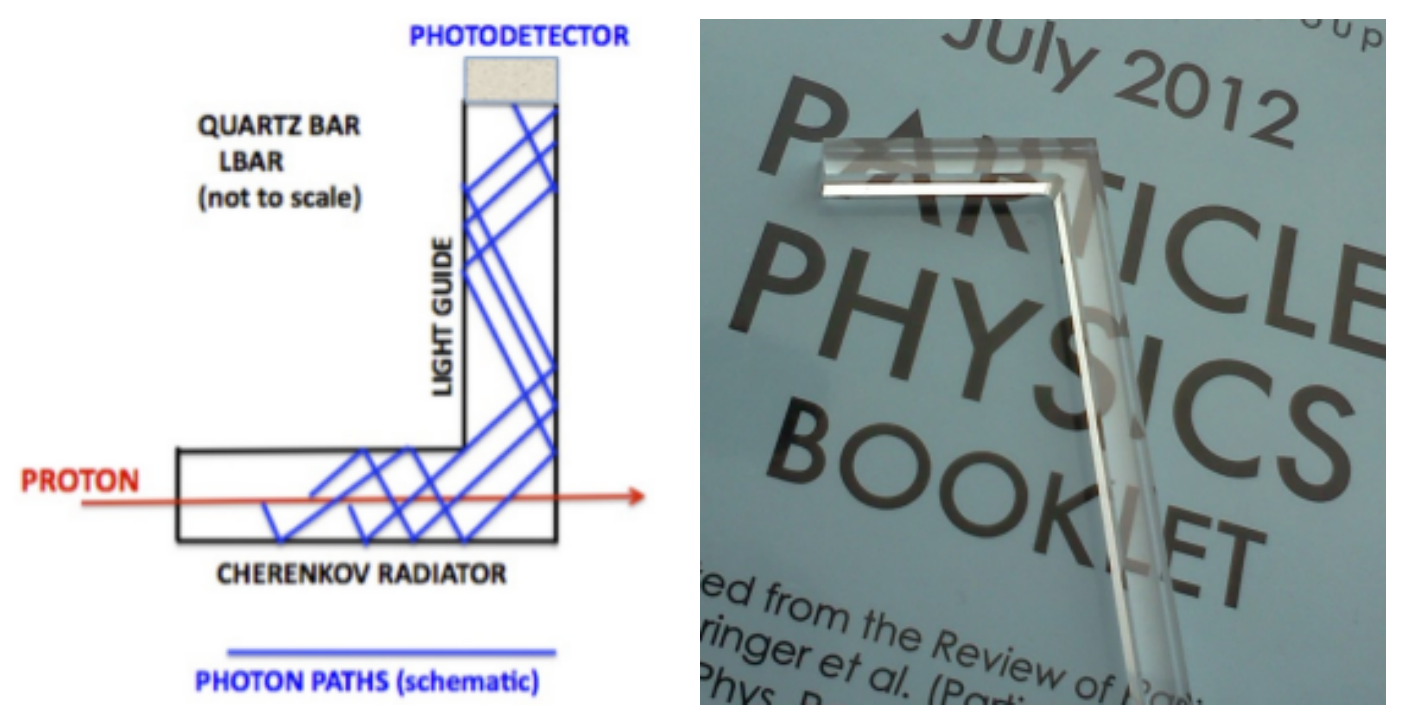

Figure 5. Cherenkov light rays in the radiator and light guide bar, schematic and not to scale (left); L-shape sapphire bar (right).

bar, which help to allow the photodetector to be far from the beam. In addition the blue light path length is less than that of the red light, unlike in the radiator bar.

The beam test of the QUARTIC prototype was tested with $120 \mathrm{GeV} / \mathrm{c}$ proton beam in FNAL. We measured the signals from four bars in-line in 5 GSPS waveform digitisers DRS4 [12], together with the signal from a faster $(\approx 8$ ps resolution) PHOTEK PMT240 [13] behind the test modules. We sent the $2 \times 2 \mathrm{~mm}^{2}$ beam through four $30 \mathrm{~mm}$ (short) radiator bars (with $40 \mathrm{~mm}$ light guide bars) and separately through four $40 \mathrm{~mm}$ (long) bars ( $43 \mathrm{~mm}$ LG bar). Figure 6 shows a typical vent; the green (lowest) trace is the PMT240 signal $(50 \mathrm{mV} /$ division), and the other traces are the signals from three $30 \mathrm{~mm}$ bars, with $2 \mathrm{~ns} / \mathrm{div}$ and $20 \mathrm{mV}$ per division. The time resolution for a single bar is $\approx 30 \mathrm{ps}$, in agreement with Monte-Carlo simulations. 

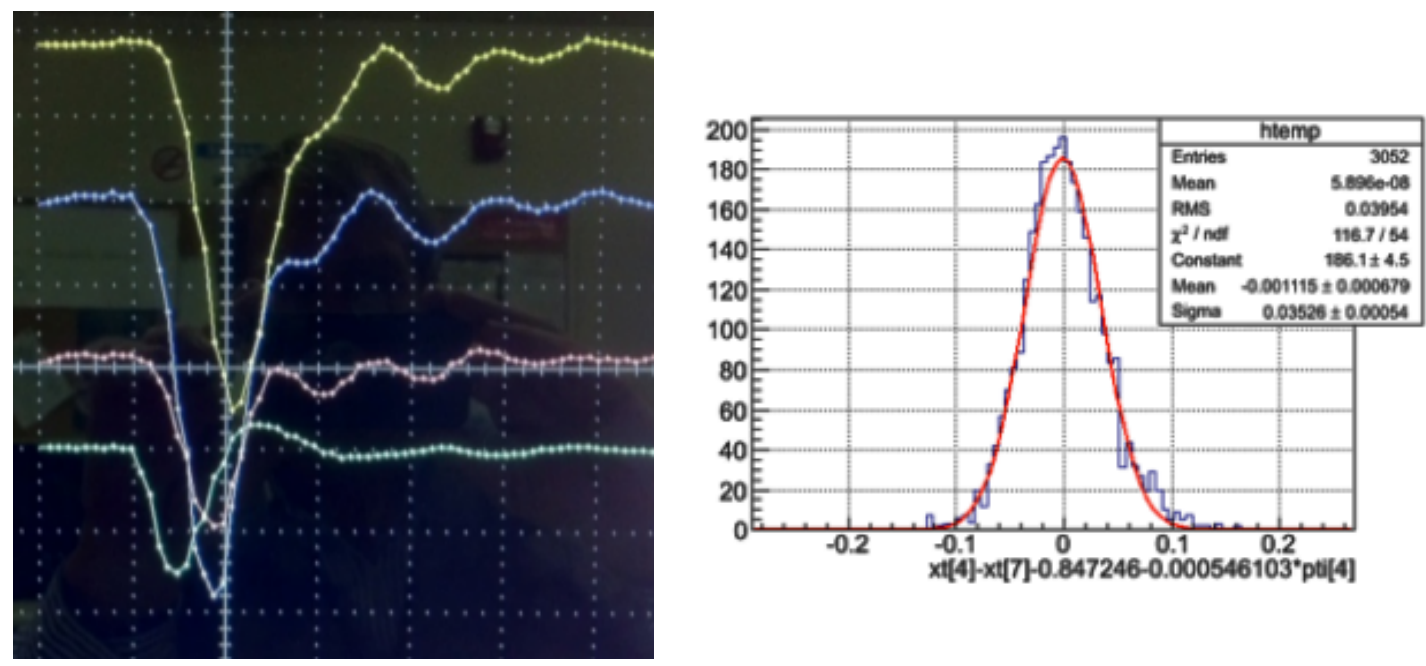

Figure 6. Waveforms in the DRS-4 of one proton through three L-bars and the PMT240 (bottom trace). The time scale is $2 \mathrm{~ns} / \mathrm{div}$ and the vertical scale $20 \mathrm{mV} / \mathrm{div}$ for the top three traces (SiPM's) and $50 \mathrm{mV} / \mathrm{div}$ for the bottom trace (reference PMT240) (left); the time difference between one L-bar (30 mm radiator, $40 \mathrm{~mm}$ light guide, Hamamatsu MPPC type S10362-330050C [14]) and the reference time signal (PMT240 in beam) (right).

Other improvements are in using the other radiator and photoreadout. Sapphire $\left(\mathrm{Al}_{2} \mathrm{O}_{3}\right)$ is promising radiator material, with a higher refractive index, $(n=1.70$ at $400 \mathrm{~nm})$ resulting in more Cherenkov photons that quartz. However the time spread over a bar of given radiator length goes like $n^{2} / c$, and the dispersion is higher. GEANT4 [15] simulations show a $30 \%$ improvement in the time resolution. Technological problems with the production of L-shape sapphire bar has been solved succesfully [16] and the bar shown in figure 5. The silicon PM are used as photoreadout for individual channels. The measurements with new configuration are in progress now.

\section{Conclusion}

At the LHC, diffractive cross sections can be measured with the addition of FSC to the present CMS or ATLAS detectors to cover the lowest diffractive masses, below $\approx 5 \mathrm{GeV}$. With the proposed detector arrangement, important new data can be obtained by tagging single and central diffractive processes. The efficiency of the FSC system for detecting rapidity gaps is shown to be adequate for the proposed studies of single- and central-diffraction.

We have developed Cherenkov counters using quartz bar radiators and both MCP-PMT and SiPM readout, designed to measure the time of protons at the LHC very close to the beam, with resolution $\sigma_{t} \approx 10 \mathrm{ps}$. The area required is only $\approx 2 \mathrm{~cm}^{2}$. Our latest design, with a novel L-bar geometry, has $\sigma_{t}=16 \mathrm{ps}$, with a path for improvement, and satisfies the other requirements of edgelessness (within about $100 \mu \mathrm{m}$ ), sufficient radiation hardness, ability to measure several protons within a bunch (time spread $\sigma_{b}=150 \mathrm{ps)}$ and to be active every $25 \mathrm{~ns}$ (the bunch separation). 


\section{Acknowledgments}

Some of the earlier studies were carried out in collaboration with J. Vavra (LBNL), A. Brandt (University of Texas at Arlington), J. Pinfold and Shengli Liu (Univ. Alberta). We thank also Austin Ball, Detlef Svoboda, Albert De Roeck, Krzysztof Piotrzkowski, and Mikhail Ryskin for valuable discussions.

\section{References}

[1] M. Albrow et al., Forward physics with rapidity gaps at the LHC, 2009 JINST 4 P10001.

[2] K.G. Boreskov, A.B. Kaidalov and O.V. Kancheli, Strong interactions at high energies in the Reggeon approach, Phys. Atom. Nucl. 69 (2006) 1765.

[3] M.G. Ryskin, A.D. Martin, V.A. Khoze and A.G. Shuvaev, Soft physics at the LHC, J. Phys. G 36 (2009) 093001 [arXiv: 0907. 1374].

[4] TOTEM collaboration, G. Anelli et al., The TOTEM experiment at the CERN Large Hadron Collider, 2008 JINST 3 S08007.

[5] CMS collaboration, Physics and beam monitoring with forward shower counters (FSC) in CMS, CMS-NOTE-2010-015 (2010).

[6] S.I. Parker, C.J. Kenney and J. Segal, 3D - a proposed new architecture for solid-state radiation detectors, Nucl. Instrum. Meth. A 395 (1997) 328.

[7] ATLAS IBL collaboration, ATLAS insertable B-layer technical design report, CERN-LHCC-2010-013, ATLAS-TDR-19 (2010).

[8] http://www.eljentechnology.com/.

[9] F. Alessio, Beam, background and luminosity monitoring in LHCb and upgrade of the LHCb fast readout control, CERN-THESIS-2011-047 (2011).

[10] M.G. Albrow and A. Rostovtsev, Searching for the Higgs boson at hadron colliders using the missing mass method, hep-ph/0009336.

[11] M.G. Albrow et al., Quartz Cherenkov counters for fast timing: QUARTIC, 2012 JINST 7 P10027.

[12] S. Ritt, Domino Ring Sampler, DRS4 evaluation board manual, http://drs.web.psi.ch/.

[13] http://www.photek.com/.

[14] http://www.hamamatsu.com.

[15] S. Agostinelli et al., GEANT4 - a simulation toolkit, Nucl. Instrum. Meth. A 506 (2003) 250; http://geant4.cern.ch/.

[16] http://alfa-tm.com. 